\title{
Estudo refracional em crianças pseudofácicas
}

\author{
Refraction studyon pseudophakicchildren
}

\author{
Gerson López Moreno ${ }^{1}$ \\ Márcia Beatriz Tartarella ${ }^{2}$ \\ Fernando Antonio de Macedo Leal ${ }^{3}$
}

\begin{tabular}{|c|}
\hline RESUMO \\
\hline $\begin{array}{l}\text { Objetivo: Verificar as alterações refratométricas em olhos de crianças sub- } \\
\text { metidas à cirurgia de catarata com implante de lente intra-ocular (LIO) e } \\
\text { pesquisar a previsão da biometria nesses casos. Métodos: Foram estudados } \\
33 \text { olhos com medição prévia da acuidade visual com indicação cirúrgica de } \\
\text { catarata com implante de lente intra-ocular no Ambulatório de Catarata } \\
\text { Congênita daEscolaPaulista de Medicina- UniversidadeFederal deSãoPaulo } \\
\text { (UNIFESP-EPM), desde janeiro de } 1998 \text { até dezembro de } 2000 \text {. No pós- } \\
\text { operatório foi realizada a refração, prescritos os óculos com o adequado } \\
\text { tratamento para estimulação visual da criança e a medida da acuidade visual } \\
\text { final comacorreção. Foramanalisadas: refrações finais, aacurácia dabiometria } \\
\text { e os possíveis fatores de erros. Resultados: Foram realizadas } 33 \text { cirurgias em } \\
29 \text { crianças. Oseguimento teve média de } 10,15 \text { meses com variação de } 2 \text { até } 34 \\
\pm 8,18 \text { meses. Em } 43 \% \text { dos casos obteve-se visão igual ou maior que } 20 / 50 \text {. } \\
\text { Quarentae seis porcento dos pacientes apresentaram refraçãofinalentre+1,00 } \\
\text { e -1,00 dioptrias esféricas. Conclusão: O tratamento da catarata congênita } \\
\text { mediante implante de lente intra-ocular continua controverso, sendo que os } \\
\text { resultados a longo prazo não são conhecidos. Número grande de pacientes } \\
\text { foram operados tardiamente evoluindocom ambliopia baixa acuidade visual } \\
\text { mesmo após o tratamento devido a grande dificuldade de acesso ao tratamento } \\
\text { adequado e precoce. Apesar da refração residual os pacientes obtiveram boa } \\
\text { acuidade visual. Em } 21 \% \text { dos casos que permaneceram com alteração refrato- } \\
\text { métrica com hipermetropia ainda terão aumento no seu diâmetro axial e } \\
\text { conseqüentemente miopização com redução de sua refração. }\end{array}$ \\
\hline
\end{tabular}

Descritores: Catarata/congênito; Extração de catarata; Implante de lente intra-ocular; Acuidade Visual; Refração ocular; Biometria, Criança

\section{INTRODUÇÃO}

O implante de lente intra-ocular (LIO) em crianças vêm sendo utilizado com mais freqüência, com a finalidade de melhorar a acuidade visual e evitar ambliopia, tudo isto com o desenvolvimento de novas técnicas cirúrgicas como a facoaspiração e o desenvolvimento de novos materiais das lentes intra-oculares, que apesar de tudo isto a cirurgia de catarata em crianças continua sendo um grande desafio para a oftalmologia pelas complicações que podem ocorrer ${ }^{(1)}$. O objetivo deste trabalho é verificar as alterações refratométricas nos olhos de crianças submetidas à cirurgia de catarata com implante de lentes intra-oculares ${ }^{(2-3)}$.

\section{MÉTODOS}

Foram estudados 33 olhos com indicação cirúrgica de catarata com implante de (LIO) no ambulatório de catarata congênita da Escola Paulista 
de Medicina, Universidade Federal de São Paulo, desde janeiro de 1998 até dezembro de 2000. Todos apresentavam acuidade visual menor que 20/50.

Os cálculos ecobiométricos das LIOs foram realizados aplicando-se as fórmulas Hoffer-Q em olhos menores que $22 \mathrm{~mm}$ e SRK-T em olhos maiores que $24,5 \mathrm{~mm}^{(4-5)}$. Utilizou-se a LIO com dioptria mais próxima ao valor encontrado para emetropia.

Todas as cirurgias foram realizadas no Hospital São Paulo sob anestesia geral.

As técnicas cirúrgicas utilizadas foram facectomia extracapsular com aspiração manual do cristalino com dupla via de Simcoe, ou facoaspiração com aparelho (Universal II-Alcon), com implante de LIO, com incisão escleral ou limbar. Em todos os casos foi usada a substância viscoelástica. As suturas foram feitas com pontos separados com fio de mononylon 10-0.

As medicações tópicas utilizadas no período pós-operatório foram colírios com antibiótico, corticóide, midriáticos e um antiiflamatório não hormonal via oral.

Posteriormente foi realizada a refração e prescritos os óculos bifocais ou multifocais ou não, com o adequado tratamento para estimulação visual da criança, e realizada a medida da acuidade visual final com correção. Foram analisadas as refrações finais, a acurácia da biometria e os possíveis fatores de erros.

\section{RESULTADOS}

Foram realizadas 33 cirurgias em 29 crianças, sendo 21 pacientes do sexo masculino e 8 do sexo feminino. A idade dos pa-

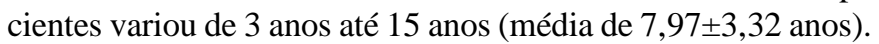

Dezenove olhos foram operados pela técnica de facectomia extracapsular e catorze olhos foram operados através da facoaspiração com implante de lente intra-ocular sempre que possível no saco. Foram implantadas 29 lentes de PMMA e 4 lentes acrílicas.

Em cinco casos ocorreu ruptura da cápsula posterior no ato intra-operatório e foi realizada vitrectomia anterior.

O seguimento variou de 2 até 34 meses com média de 10,15 $\pm 8,18$ meses.

$\mathrm{Na}$ avaliação da acuidade visual final em $43 \%$ dos casos obteve-se visão maior ou igual a 20/50. Quarenta e seis por cento dos pacientes apresentavam refração final entre $-1,00$ e $+1,00$ dioptrias esféricas, $21 \%$ apresentaram refração entre $+1,25$ e +4,50 dioptrias esféricas (Gráfico 1, 2, 3).

\section{DISCUSSÃO}

O tratamento da catarata congênita através do implante de lente intra-ocular continua controverso onde os resultados em longo prazo não são conhecidos. Os tipos, os tamanhos, os modelos e os materiais das lentes intra-oculares estão em evolução, não havendo, ainda, a lente intra-ocular ideal para crianças. Os avanços tecnológicos e a pesquisa em biocompatibilidade poderão trazer melhores resultados ${ }^{(6-8)}$.

No estudo destes 29 pacientes foram avaliados os resulta-

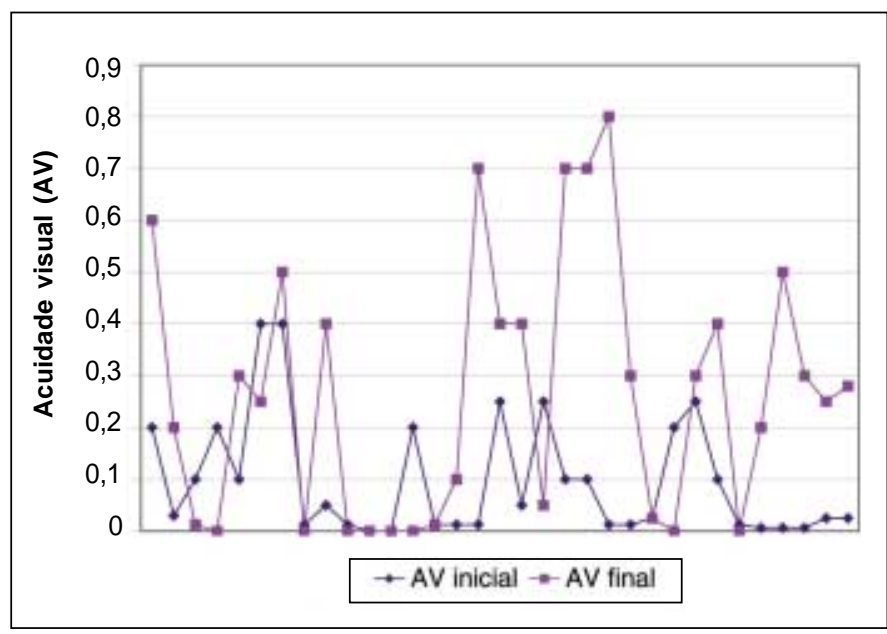

Gráfico 1 - Acuidade visual antes e depois da cirurgia com correção

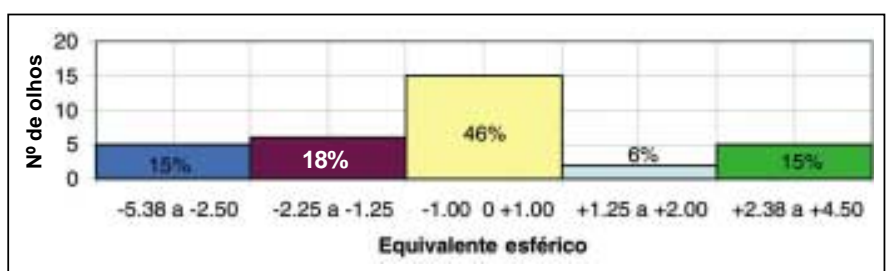

Gráfico 2 - Refração final (Equivalente esférico)

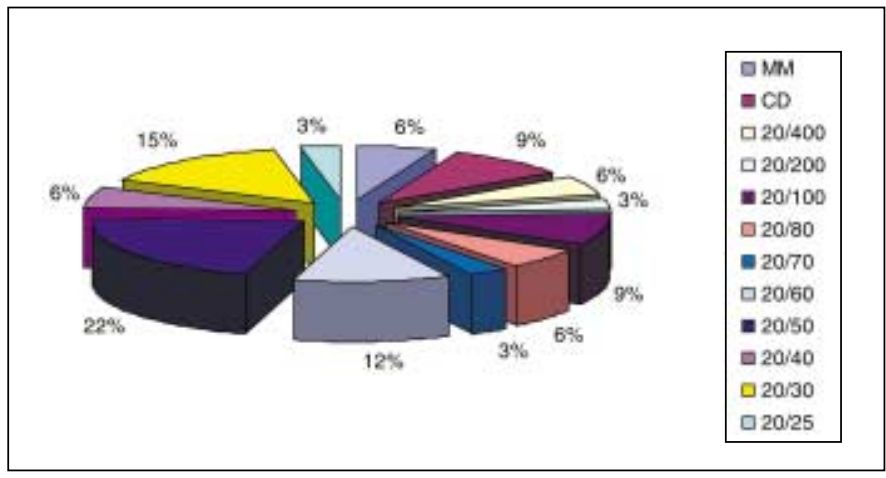

Gráfico 3 - Acuidade visual final

dos refracionais em crianças em um centro universitário de treinamento e pesquisa.

Os 33 olhos foram operados por técnicas de aspiração manual ou mecanizado (facoemulsificador), sendo na maioria dos casos núcleos moles. Pela elasticidade em crianças existe uma maior instabilidade do segmento anterior durante o ato cirúrgico dificultando a capsulorrexis anterior e o implante da LIO no saco capsular. Um número grande de pacientes foi operado tardiamente, evoluindo com ambliopia e baixa acuidade visual, devido a grande dificuldade de acesso ao tratamento adequado e precoce.

Como se pode observar no gráfico 1 , a acuidade visual dos pacientes melhorou em sua grande maioria, com exceção dos 
pacientes que já tinham diagnóstico de ambliopia. Quarenta e três por cento dos pacientes alcançaram acuidade visual de 20150 ou mais com a melhor correção, o que demonstra um bom resultado visual para estas crianças que têm uma expectativa de vida longa.

A LIO implantada nestes olhos foi utilizada conforme os cálculos de biometria para emetropia. Mesmo assim observaram-se resíduos refracionais nestes pacientes.

Em $46 \%$ dos olhos a refração ficou próxima à emetropia (de $-1,00$ até $+1,00$ equivalente esférico).

Nos $21 \%$ de olhos que ficaram com equivalente esférico entre $+1,25$ e $+4,38$ dioptrias esféricas, espera-se que ocorra uma emetropização, pois são olhos que estão em crescimento e ainda apresentarão miopização.

Porém nos $33 \%$ de olhos com resultado refracional de miopia poderão ter um acréscimo no seu grau de acordo com a idade em que foram operados.

Apesar dos problemas inerentes ao tratamento da catarata congênita e da pouca colaboração das crianças para a realização dos exames e da biometria, obteve-se um bom resultado refracional e visual final.

\section{CONCLUSÃO}

Que a biometria utilizada foi adequada em $46 \%$ dos olhos, mantém-se uma refração final próxima da emetropia.

Novos estudos necessitam ser realizados para obter-se uma melhor acurácia na biometria dos olhos de crianças portadoras de catarata congênita.

\section{ABSTRACT}

Purpose: To verify the refractive alterations in eyes of children submitted to surgery with implant of intraocular lens (IOL) and to investigate prediction of biometry in these cases. Methods: Thirty-three eyes were studied with previous measurement of visual acuity with indication for cataract surgery with implant of intraocular lens in the Outpatient Clinic of Congenital Cataract of the Federal of University of São Paulo/ Paulista School of Medicine, (UNIFESP-EPM) from January, 1998 to December, 2000. After the surgery, refraction, pres- cription of glasses with appropriate treatment for visual stimulation of the children, and measurement of the final corrected visual acuity were performed. Final refraction, biometric accuracy and possible error factors were analyzed. Results: Thirty-three surgeries in 29 children were performed. Mean followup time was 10.15 months ranging from 2 to $34 \pm 8.18$ months. In $43 \%$ of the cases a vision equal to or grater than $20 / 50$ was obtained. Forty-six percent of the patients presented a final refraction between +1.00 and -1.00 spherical diopters. Conclusion: Treatment of congenital cataract through implant of intraocular lens continues to be controversial, where longterm results are not known. A great number of patients were operated on at a late time and progressed with amblyopia and low visual acuity even after treatment with difficult access to early and adequate treatment. In spite of residual refraction the patients obtained good visual acuity. Twenty-one percent of the cases with refractive alteration with hyperopia will still have increase in their axial diameter and consequently myopia with reduction of their refraction.

Keywords: Cataract/congenital; Cataract extraction; Lens, implantation, intraocular lens; Visual acuity; Refraction, ocular refraction; Biometry; Child

\section{REFERÊNCIAS}

1. Tartarella MB, Kawakami LT, Scarpi MJ, Hayashi S. Aspectos cirúrgicos em catarata congênita. Arq Bras Oftalmol. 1995;58(1):24-8.

2. Wilson ME, Saunders RA, Roberts EL, Apple DJ. Mechanized anterior capsulectomy as an alternative to manual capsulorhexis in children undergoing intraocular lens implantation. J Pediatr Ophthalmol Strabismus. 1996;33(4): 237-40. Comment in: J Pediatr Ophthalmol Strabismus. 1997;34(4):210-1.

3. Lambert SR, Drack AV. Infantile cataracts. Surv Ophthalmol. 1996;40(6): 427-58. Comment in: Surv Ophthalmol. 1997;41(4):357.

4. Tromans C, Haigh PM, Biswas S, Lloyd IC. Accuracy of intraocular lens power calculation in pediatric cataract surgery. Br J Ophthalmol. 2001;85(8): 939-41.

5. Gimbel HV. Posterior continuos curvilinear capsulorhexis and optic capture of the intraocular lens to prevent secondary opacification in pediatric cataract surgery. J. Cataract Refract Surg. 1997;23(Suppl 1):652-6.

6. Benezra D, Cohen E. Posterior capsulectomy in pediatric cataract surgery: the necessity of choice. Ophthalmology. 1997;104(12):2168-74.

7. Ventura M, Delgado AC, Ventura LO, Tito IR, Endris D. Facoemulsificação/ aspiração e implante primário de lente intra-ocular dobrável de acrílico em 28 olhos com catarata infantil. Rev Bras Oftalmol. 2001;60(1):33-9.

8. Plager DA, Kipfer H, Sprunger DT, Sondhi N, Neely DE. Refractive change in pediatric pseudophakia: 6-year follow-up. J Cataract Refract Surg. 2002;28(5): $810-5$ 